\title{
Self-organizing Systems in the Light of the Arrows of Orderedness, Symmetry, and Entropy
}

\author{
György Darvas
}

Institute for Research Organization of the Hungarian Academy of Sciences and Symmetrion 18 Nádor St., Budapest, H-1051 Hungary (P. O.B. 994, Budapest, H-1245 Hungary) darvasg@helka.iif.hu

\begin{abstract}
The paper makes an attempt to resolve two conceptual mingling: (a) the mingling of the two interpretations of the concept of orderedness applied in statistical thermodynamics and in symmetrology, and (b) the mingling of two interpretations of evolution applied in global and local processes. In conclusion, it formulates a new interpretation on the relation of the emergence of new material qualities in selforganizing processes on the one hand, and the evolution of the universe, on the other. The process of evolution is a sequence of emergence of new material qualities by self-organization processes, which happen in negligible small segments of the universe. Although thermodynamics looks at the universe as a closed (isolated) system, this holds for its outside boundaries only, while the universe has many subsystems inside, which are
\end{abstract}

not isolated (closed), since they are in a permanent exchange of matter, energy, etc. with their environment (with the rest of the universe) through their open boundaries. Any "emergence" takes place, i.e., all new qualities come into being just in these small open segments of the universe. The conditions to apply the second law of thermodynamics are not present here. Therefore, global evolution of the universe is the consequence of local symmetry decreases, local decreases of orderedness, and possible local decreases of entropy.

Keywords: emergence, self-organization, arrows of time, symmetry, entropy, orderedness, evolution, ontological levels, global- and local processes

\section{Symmetries of Simple and Compound Systems}

Any system has a certain number of symmetries. For example, a regular triangle has three symmetry axes and a 3-fold rotational symmetry. 


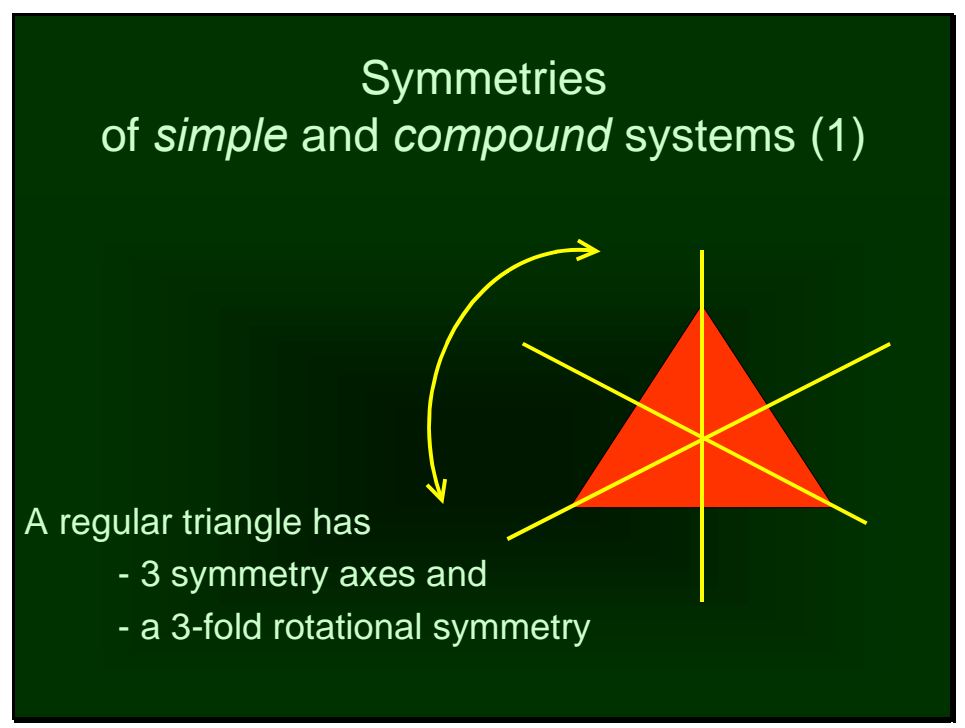

Figure 1

As constituents of a compound system, the same systems may lose a few of their symmetries, which they have owned as individuals. For example, the regular triangle shown in Figure 1 may compose a system together with 3 further ones (cf., Figure 2), and as constituents of this compound system they preserve only one symmetry axis each.

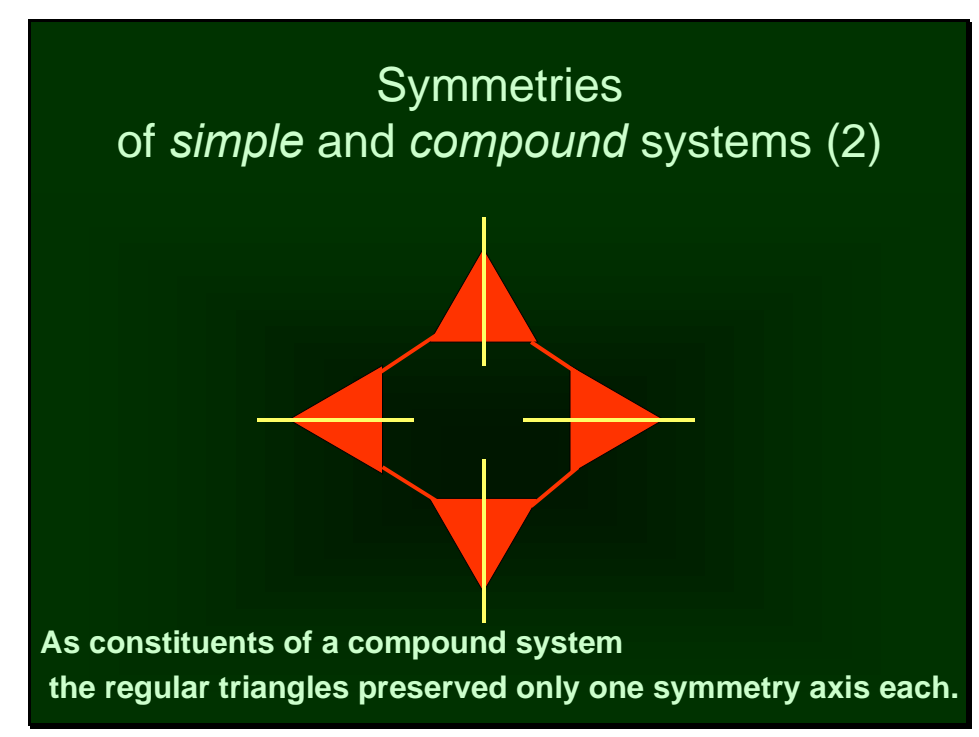

Figure 2

However, there appear new symmetries in the compound system - cf., the symmetry axes of the square formed by the four triangles.

This simple example is subject of a more general set of laws - in particular mainly the (2.2 a-d) below namely, the laws of ontological levels and symmetry breaking discussed in more detail in Darvas (1998a, 1998b). We quote here simply the laws, omitting their detailed explanation.

\section{The Laws of Symmetry Breaking}




\subsection{The Law of the Determining Role of Lower Levels}

(2.1a) Among two consecutive (a lower and an upper) levels, the lower level potentially (but only potentially) possesses the characteristic type of interaction of the consecutive upper level; i.e., that the preceding lower level's types of interaction play the determining role in the development and existence of any level's characteristic interaction. However,

(2.1b) In the interrelation of two different (an upper and a lower) levels, generally the upper level's structure affects actively the other, since

(2.1c) Any lower level material structure can reflect its environment only on its own (lower) quality and own level. Within that, the material structure of a lower level can reflect the material structures corresponding to the upper level's forms of material motion also only on its own (lower) level.

(The two statements in (2.1c) are not-certainly equivalent, because the given levels are determined per definitionem by their characteristic interaction and not by the corresponding form of material motion.) For example, any inanimate being can reflect an animal only as a physical object, and cannot reflect its biological properties; no animal can discern the social differences between human beings.

Since the relation of the two (lower and upper) levels is not symmetric, this law does not open the door to any reductionism. A reductionist approach would allow only the following kind of statement, viz., "among two consecutive levels, the lower level possesses the characteristic type of interaction of the consecutive upper level". Nevertheless, according to these laws, (2.1a) limits the existence of the upper level's characteristic interaction at the lower levels to potentiality, while (2.1b) and (2.1c) together contradict any statement which denies the appearance of new qualities at the upper levels. The way is open for the emergence of new material qualities, known also as self-organization of matter.

(2.2) The law of correspondence between the ontological levels and their potential symmetry properties.

(2.2a) Each qualitatively higher organizational form in the evolution of matter is marked by the loss of a certain symmetry property, and

(2.2b) Each loss of a potential symmetry property of matter traces a new material quality.

Consequently, the precondition of the development (in its relative totality) of a qualitatively new (material) level is the breaking of a certain symmetry (property), and at the same time, the condition of the continuance (existence) of the new level is to possess (new) conserved properties. Therefore

(2.2c) Parallel with the appearance of new material qualities and new (higher) ontological levels, there appear also new symmetries.

(2.2d) These new symmetries qualitatively differ from those that existed at the preceding (lower) levels and that have been broken at the given level. These new symmetries involve new conserved properties.

As a conclusion, the lower and higher ontological levels can be traced by a sequence of symmetry breakings. Thus it can be formulated, that

(2.3) Each symmetry breaking leads to a higher organizational level of matter.

(2.4) Each higher organizational level of matter is - in a certain sense - less stable than the preceding one.

The cited set of laws marks the arrow of self-organizing processes in matter. As a convention, it is accepted as the arrow of evolution. To go beyond the convention, we should make certain restrictions. This will be one of the subjects of this paper. 


\section{The Arrow of Time}

According to the mentioned convention, the arrow of evolution coincides with the arrow of time ${ }^{1}$. This follows from the conditions of the Second Law of Thermodynamics, according to which (a) in a closed system, where (b) no phase transitions take place, the physical processes evolve in one direction. ${ }^{2}$ When referring to the "Second Law" one should keep in mind the conditions (a) and (b) of its applicability.

\section{Symmetry and Orderedness}

According to the law (2.2a) a system evolves in the direction where its symmetry decreases. Constituents of a symmetric system are ordered. The more its constituents are ordered, the more symmetric is the given system, and vice versa. When the system's symmetry decreases, there will be a decrease of its orderedness too.

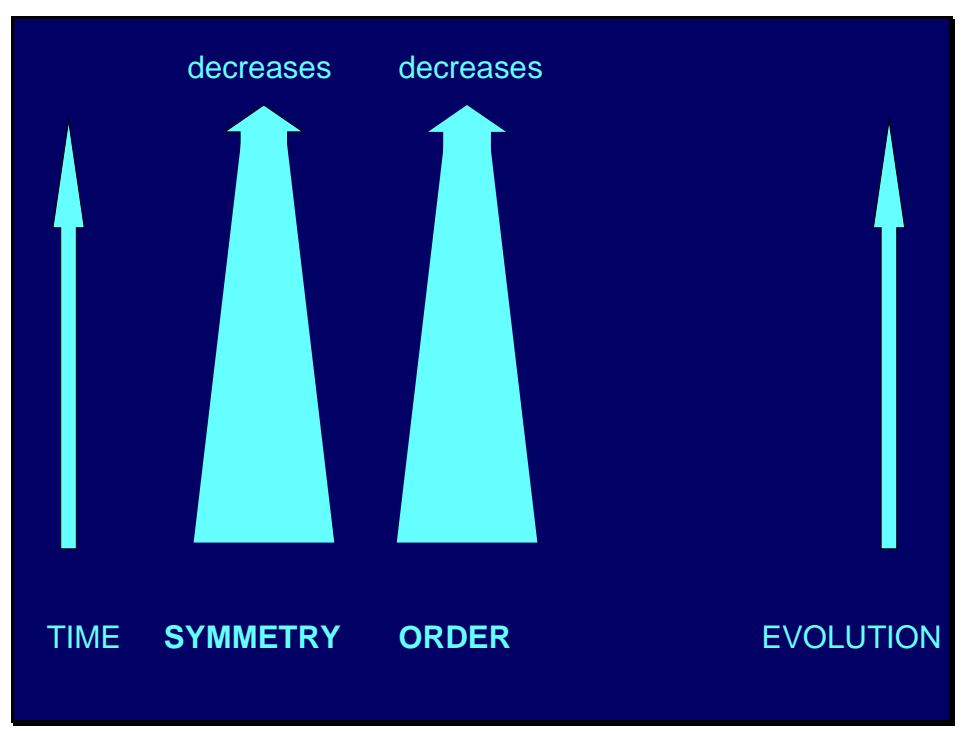

Figure 3

\footnotetext{
${ }^{1}$ Arrow of time. Time is heading in one direction - at least in global terms. Locally one can speak about time-reversed processes. What does this mean? This means, that certain physical laws allow reversible processes. More precisely, if we can record the consecutive physical states $\psi_{A}, \psi_{B}$ and $\psi_{C}$, at the moments $t_{A}, t_{B}$, and $t_{C}$, where $t_{A}<t_{B}<t_{C}$, then we may observe the process $\psi_{C} \rightarrow$ $\psi_{B} \rightarrow \psi_{A}$ at the moments $t_{C}^{\prime}, t_{B}^{\prime}$, and $t_{A}^{\prime}$, where $t_{C}^{\prime}<t_{B}^{\prime}<t_{A}^{\prime}$. In the latter case, the clock assigned to the measuring instrument paces counterwise the clock on the wall of the lab, i.e., a larger system. That means, a 'reversed' time always postulates the existence of a 'lab' time (or 'global' time), compared to which its direction is reversed. We can choose from among the following two statements: either our process is the same in both cases and the local time (in which it took place) was reversed, or we state, that we observed two opposite processes compared to the same, global time. Compared to the other processes observed in the same $\mathrm{lab}$, it seems more correct to insist on the first statement, while in philosophical terms the latter seems more acceptable.
}

${ }^{2}$ In accordance with Boltzmann's approach, this direction does not depend on whether the investigated system is expanding or contracting, and whether it is subject of further physical interactions. Nevertheless, Ne'eman (2003, and already in 1969, 1970, Aharony and Ne'eman 1970a, 1970b) identified seven time-arrows, connected to five different physical and two non-physical phenomena, which can define independently the flow of time. Among these seven there is one the thermodynamical arrow, one connected with the evolutionary drive (in philosophical sense, including everything from cosmogony to epistemology), one with the universal expansion, one with the radiation and the advanced or retarded potentials, one with the CPT conservation (resulting from the dependence of simultaneous CP- and T-violations), one is the gravitational arrow (the action leading to the formation of black holes), and one is the cognitive inner human sense of duration. This paper treats the relation of the first listed two arrows of time. 


\section{Orderedness and Entropy}

According to Boltzmann's formulation of the second law of thermodynamics, when a system evolves in the direction of statistically lessening order of its constituents, its entropy increases. And usually systems evolve towards lessening order of their constituents.

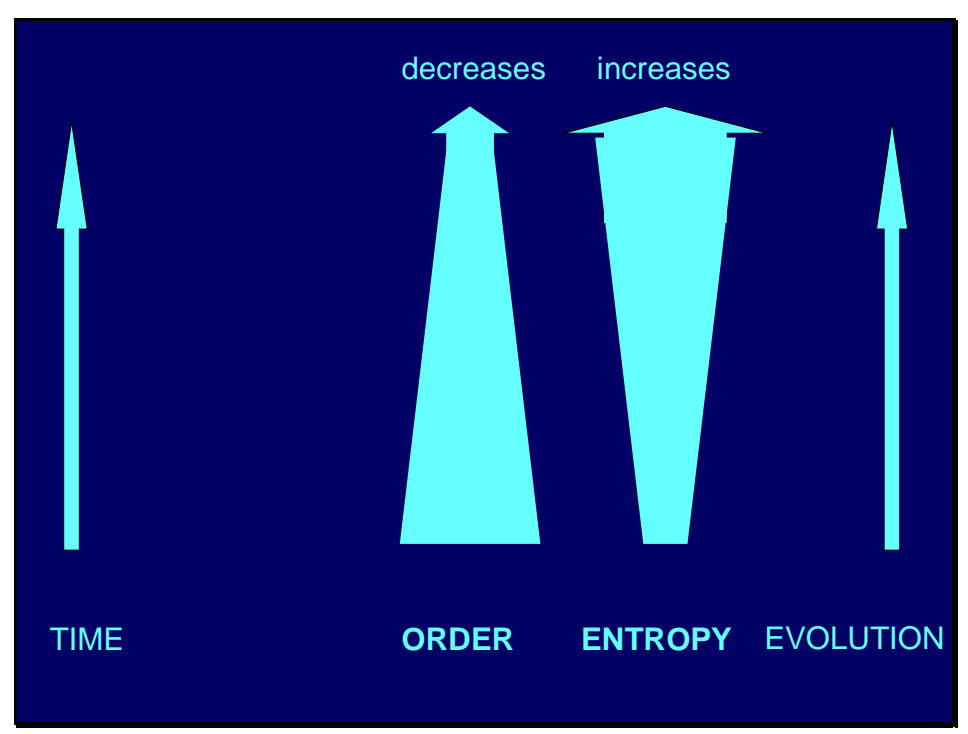

Figure 4

\section{Symmetry, Order and Entropy: Conceptual Differences in Symmetrology and Statistical Thermodynamics}

Let's compare, what we have stated on the relation of the arrows of time, evolution, symmetry and orderedness, as well as of the arrows of time, evolution, entropy and orderedness:

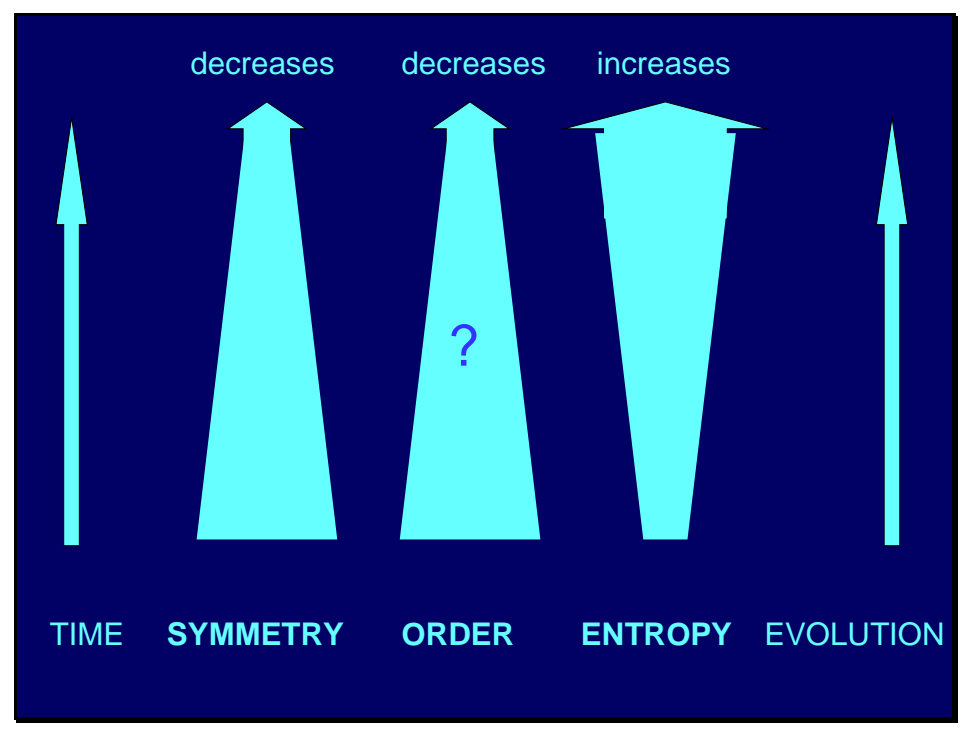

Figure 5

The arrows of the five properties seemingly coincide in the two statements. Nevertheless, we should question the arrow of the orderedness. Why? 
There is a difference in the interpretation of the 'orderedness' in the compared two cases. Let's analyse, when do we speak about a 'more ordered' and a 'less ordered' system? We will show, that we use this term in different meanings according to our symmetry concept and in statistical thermodynamics.

Our concept on the relation of symmetry and orderedness has its roots in crystallography. According to this, the most ordered state of a crystal is, when its all constituents are placed in equal distances (and their vector properties are headed in the same direction). A crystal represents certain symmetry, thanks to the order among its constituents. In these terms glasses - or gas, filling a box - represent less symmetric and less ordered (i.e., more chaotic) systems.

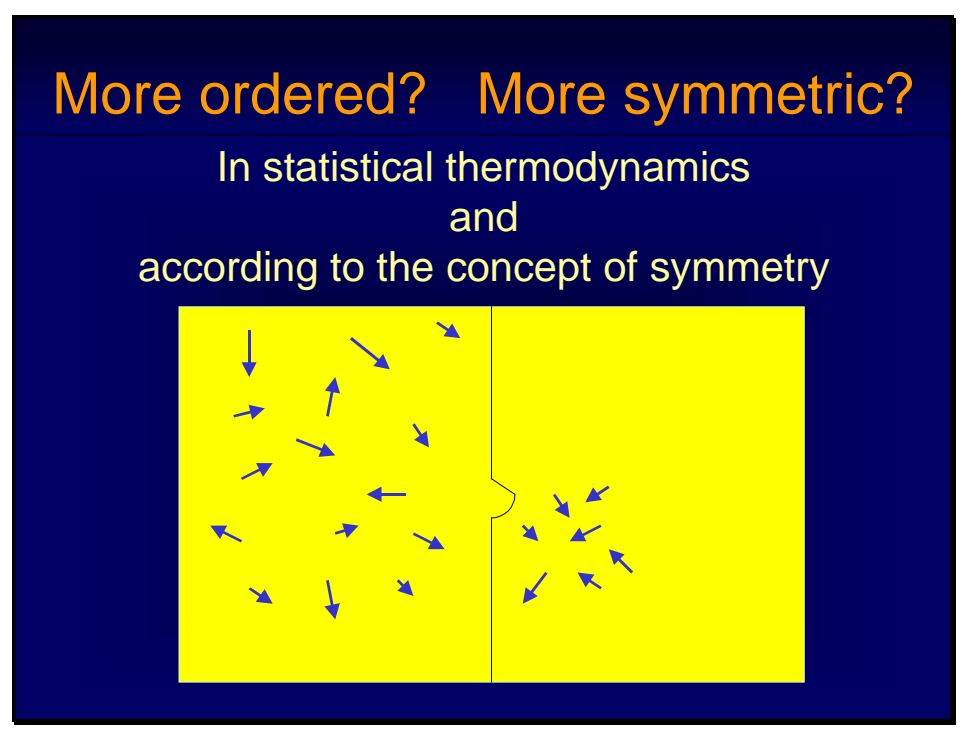

Figure 6

According to statistical thermodynamics, gas molecules filled in a segment of a box (right box of Fig. 6) represent a more ordered state. When the system is left alone, and the molecules of the gas fill the box almost equidistantly (left box of Fig. 6), that will be interpreted as a less ordered state of the system.

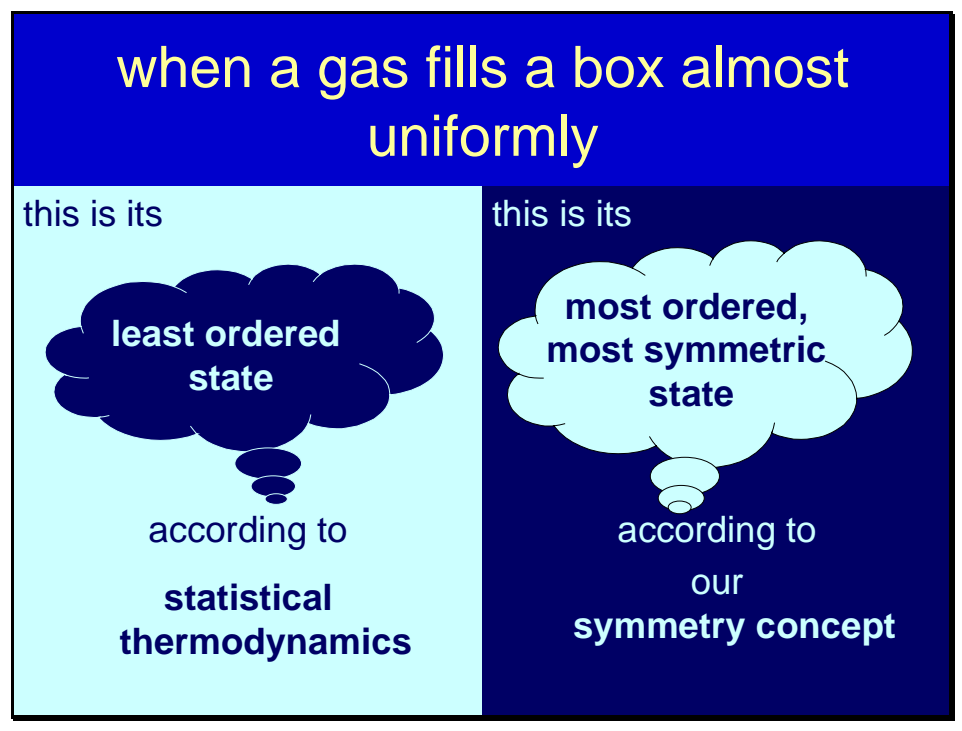

Figure 7 


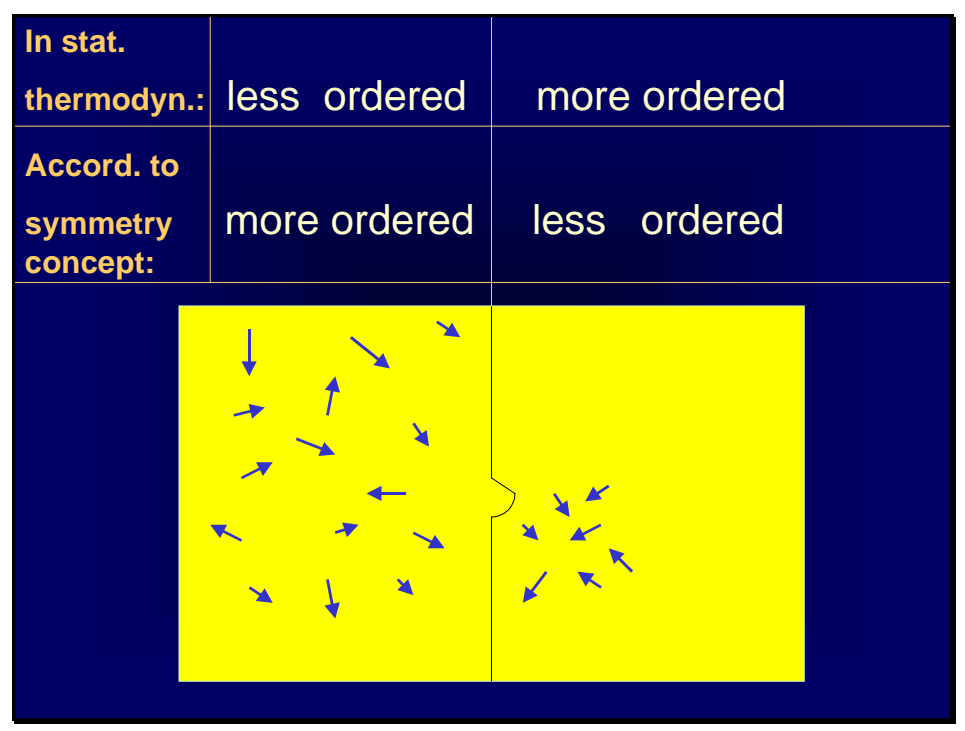

Figure 8

These contradicting statements hold because we had not used the terms in the same meaning and yet, the table in Figure 5 showed a concordant coincidence of the arrows. How could it happen? There should be something else, something that me must also take into account.

\section{Self-organizing Processes and Emergence of Higher Hierarchical Levels}

When a process of phase transition, or emergence of a new material quality takes place in a small segment inside the system, that part of the system will form an open subsystem of the larger one. Such a situation exists, for example, when a few atoms form a molecule, etc. This small segment of the system will no more be closed, and the whole system will no more be homogeneous (in qualitative terms). The larger system can - in principle - be widened to get identified with the universe. Emergence takes place always in such local environments that represent a relatively negligible volume compared to the universe. Such emergence takes place - in certain conditions - when a new quality is being formed spontaneously. This is the process of self-organization. A higher hierarchical level is under formation locally ${ }^{3}$, within the wider, global system.

\footnotetext{
${ }^{3}$ Locality will be meant in this paper not as a space-coordinate dependent property, rather as one interpreted in a given segment (environment), being relatively small compared to the universe. Global properties will be interpreted in the universe, or at least in closed systems, large enough compared to the number and extent of the entities filling it.
} 


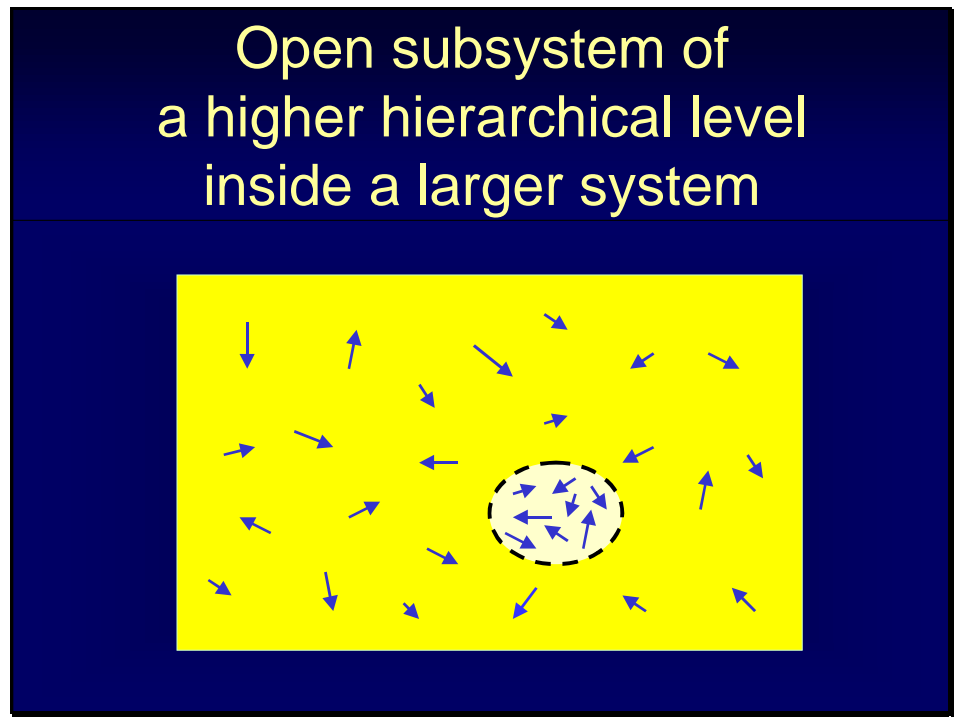

Figure 9

Note that all processes of evolution are a sequence of emergence of new material qualities by selforganization processes. These processes happen locally in open subsystems, allowing several interactions, including energy exchange, with the surrounding system. These processes happen in negligible small segments (local inner environments) of the global system.

\section{The Interpretation of Symmetry and Entropy in Global and Local Processes}

When we speak of the evolution of a thermodynamic system, we consider a closed, global system, where we neglect any possible process in small segments, any possible emergence, and the possible appearance of any new phase. We consider only a single hierarchical level from among possible material qualities. In this global thermodynamic system entropy increases, order decreases, and symmetry increases.

In the same global system in symmetrological terms entropy and symmetry would increase, and - as we showed above - order would also increase.

Let's investigate the local, higher hierarchical level, where self-organization proceeds. In symmetrological terms locally order will decrease, and so will symmetry. Since this is an open system in interaction and energy exchange with its surrounding environment, entropy may locally decrease.

In the same local open system, in thermodynamic terms order would be considered as increasing, symmetry as decreasing, and - on the same reasons as above - entropy may decrease.

These were four potential conceptual options. However, there are only two of them, which seems to be realistic. We generally speak about global processes in statistical thermodynamic terms, and about local processes in symmetrological terms. The former corresponds to the real thermodynamic processes, while the latter to the processes of self-organization. When we speak about evolution, in the global case, we understand the evolution of the given system. In the latter case we mean the evolution of matter, including the emergence of new material qualities. The two concepts are not the same. ${ }^{4}$

4 Let's look back to the Section (1), when we counted the available symmetries of the regular triangle that held for all regular triangles, globally. When we stated the decrease of the number of symmetries in the compound, four-triangles-formed system, it was held for the locally organized system, relative to the individual triangles before the emergence of the new quality. The decrease characterised the process of the emergence, and not the preceding system investigated in its qualitatively intact state. Something 
The following table shows, that the arrows of orderedness coincide in the two realisable cases, although the conceptual references are different. The arrows marked by red-framed boxes in Figure 10 show those seen in the Figures 3-5.

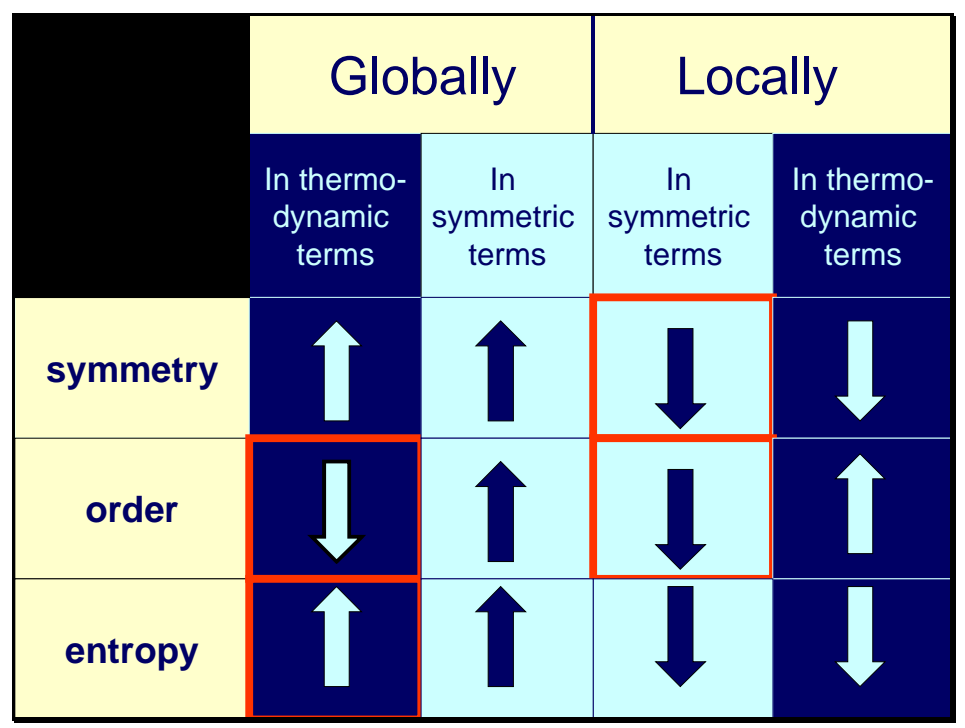

Figure 10

\section{Conclusions}

\section{1 a) Difference in Global and Local Properties}

As we saw in the table of Fig. 10, global and local properties should be interpreted in different ways. Some coincidences in the arrows shown in Fig. 5 may occur accidentally, and not by causal reasons. An example can be the interpretation of the extent of order and chaos. One could realize this situation, when we formally handled as identical the two decrease-marking arrows of order above - taken in fact from the second row of Fig. 10 - when we united Figs. 3 and 4 at the Fig. 5. Also, the arrows of symmetry and entropy may coincide; however, these coinciding directions may be opposite depending whether they were meant globally or locally. (We used the conditional 'may', because in local, open-system processes we cannot make a definite statement on the arrow of entropy, at least we should allow its decreasing value as well.)

\footnotetext{
similar could be said about the entropies of the global and the local systems. The arrow of the entropy characterises one of the systems, and not the transitions from one system to the other. Since emergence takes place in an open segment of the space (in many cases in a small-number-constituent system, not suitable to apply statistical laws for it), which is in material interaction and energy exchange with its environment, one cannot state automatically the increase of entropy. When one speaks about heat-death as a result of the evolution in the universe, one neglects this fact: entropy increase of the universe is stated for a physical universe, in which no emergence of new qualities takes place. True, emergence takes place in negligible small segments of the universe, and yet, all the evolution of the universe can be booked on the account of these processes. One speaks in thermodynamics on the evolution of physical systems, in which no phase transitions (no emergence of new material qualities) takes place - this is one interpretation of the concept of evolution. And there is another interpretation, when we take into account emergent processes too this is the evolution of the universe in philosophical terms, although with several consequences to the evolution (sequence of emergence) of the physical entities as well. The two evolution terms are marked with the same word, but they denote different concepts. One denotes processes in a single, closed system, where no emergence takes place, the other denotes transitions between two (or more) systems, representing different material qualities, and belonging to different hierarchical levels.
} 


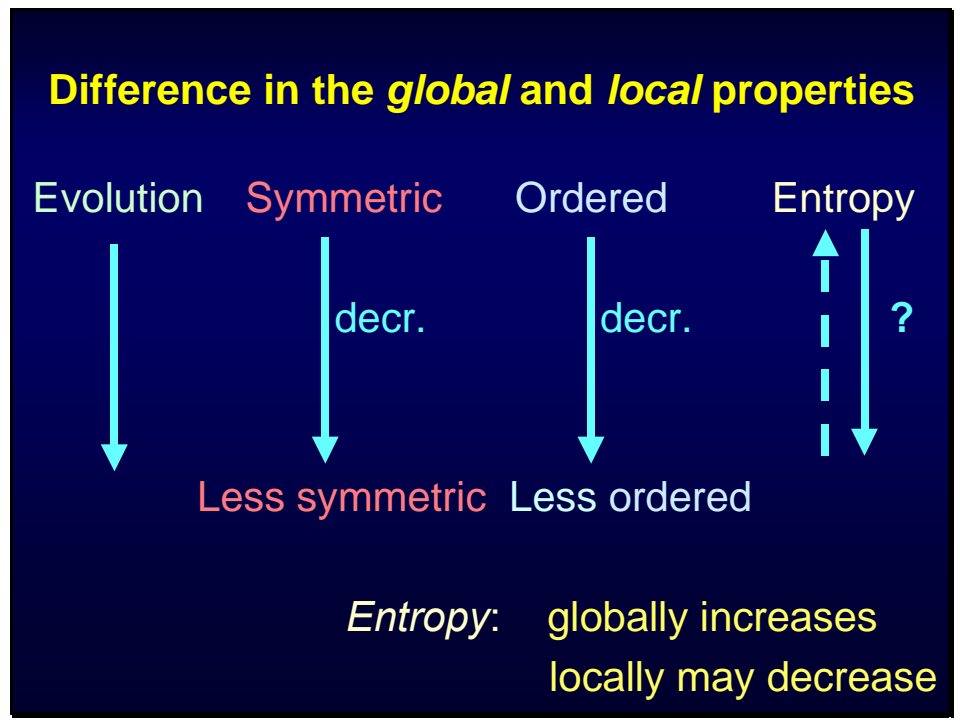

Figure 11

\section{2 b) Entropy of Self-organizing Processes}

Self-organizing processes (where new qualities come into being) happen in relatively small, open subsystems of a wider system (in which no new qualities emerge). Therefore in self-organizing processes the arrow of entropy may locally decrease. Emerging (sub)systems (and so all living systems) are such (i.e., in a non-equilibrium state, e.g., the process of protein synthesis). This fact does not contradict to the "Second Law" of thermodynamics. The second law is valid everywhere, however the conditions are not everywhere present for its functioning.

\section{3 c) The Arrows of Time and Evolution}

Evolution of the universe is the result of the described self-organizing processes. When one speaks about the evolution of the universe, one cannot neglect these small segments of space: the important processes - which play a role in the evolution of matter in the universe - take place there, and the amount of the entropy changes there. This interpretation of evolution differs from its interpretation in statistical thermodynamics, when the universe is considered as a (multi-phase) closed system, in which no phase transitions take place, therefore the emergence of new material qualities, local loss of entropy, and those small, inner, open segments (environments) at all are neglected. Note, the universe - that we have assumed in principle as closed - includes inside local, open subsystems, and thus can itself no more be considered closed as well, cf. Fig. 9.

In other terms, the process of evolution (of the universe) is a sequence of emergence of new material qualities by self-organizing processes, which happen in negligible small segments of the universe. Global evolution is a result of a sequence of local processes. Thermodynamics looks at the universe as a closed (isolated) system. The latter condition holds for its outside boundaries only. The universe has many subsystems inside. These inner subsystems have open boundaries. They are not isolated (closed), since they are in a permanent exchange of matter, energy, etc. with their environment (with the rest of the universe). Any "emergence" takes place in these small subsystems. In spite of their negligible volume compared to the universe, they cannot be neglected. All new qualities (like higher hierarchical level physical structures, biological molecules, living matter, e.g., cells, etc.) come into being just in these small segments, in these open subsystems of the universe, where one cannot apply the "Second Law" in the lack of the fulfilment of the closure/isolation condition. Small, open subsystems disappear in the sink of the universe. They are usually neglected for this reason. In fact, they cannot be neglected! A large system, whose outside boundaries are considered closed (isolated, like the physical universe) may have 
boundaries inside. These inside boundaries are open, thus the large system can no more be considered as open either. One cannot exclude the local events from the global approach.

In philosophical terms the universe "evolves" due to the existence of subsystems, where the conditions of the "Second Law" do not prevail. The arrow of the philosophically perceived evolution is directed towards the emergence of new qualities. Therefore - assuming, that no other physical process takes place simultaneously in the surrounding global system, its entropy may - at least locally - decrease. The arrow of evolution is - per definitionem - fixed to the arrow of time. Which evolution to which time? Certain physical processes can be reversed. In these cases the own clock fixed to the experiment can pace counterwise the lab's clock. Reversed time, however, always assumes a global time scale (lab's clock), compared to which it is locally reversed. Therefore, in the above statement we mean the philosophically perceived material evolution and the global time.

One can conclude that the arrow of evolution of the universe does not depend on the actual arrow of balance of entropy and that the evolution of the universe depends first of all not on the majority of the phase-transition-less physical processes that happen in the universe. Emergence of a new quality once in a minor volume of the universe may play a determining role. Global evolution of the universe is the consequence of local symmetry decreases, local decreases of orderedness, and possible local decreases of entropy (Fig. 11). Thus global evolution is determined by local events.

\section{References}

Aharony, A./Ne'eman, Y. (1970a) Time Reversal Symmetry and the Oscillating Universe. In: International Journal of Theoretical Physics, 3, pp. 437-441.

Aharony, A./Ne'eman, Y. (1970b) Time Reversal Violation and the Arrows of Time,. In: Nuovo Cimento Letters, 4, pp. $862-866$.

Arntzenius, F. (2004) Time reversal operations, representations of the Lorentz group, and the direction of time. In: Studies in History and Philosophy of Science, Part B: Studies in History and Philosophy of Modern Physics, 35, 1, pp. 31-43.

Bérczi, S./Lukács, B. (Eds.) (1993) Symmetry and Topology in Evolution. Special issues of Symmetry: Culture and Science, Vol. 4, 1-2, pp. 1-224.

Bohm, D./Vigier, J.P. (1954) Model of the causal interpretation of quantum theory in terms of irregular fluctuations. In: Physical Review, 96, 208.

Collier, J./Burch M. (1998) Order from rhythmic entrainment and the origin of levels through dissipation. In: Symmetry: Culture and Science, 9, pp. 2-4.

Darvas, G. (1987) Level theories in philosophy and physics- Exploring and interpreting the structure of matter, Vol. 5, Part 3, pp. 7679, In: 8th International Congress of Logic, Methodology, and Philosophy of Science, Abstracts, Moscow. Nauka.

Darvas, G. (1998a) Laws of symmetry breaking. In: Symmetry: Culture and Science, 9, 2-4, pp. 119-127.

Darvas, G. (1998b) Ontological levels and symmetry breaking. In: Paideia, Philosophy of Science, http://www.bu.edu/wcp/Papers/Scie/ScieDarv.htm

Darvas, G. (2003) Potential and actual time concepts, In: Buccheri, R./Saniga, M./Stuckey, W. M. (Eds.) (2003) The Nature of Time: Geometry, Physics and Perception, Dordrecht. Kluwer Academic Publishers, xvii + 446 p. pp. 417-425,

de Gortari, E. (1970) Symmetry as a scientific method. In: La symetrie. Comme principe heuristique dans les differentes sciences. Bruxelles. Office international de librairie, 135 pp. pp. 30-34

Heisenberg, W. (1971) Physics and Beyond. Encounters and Conversations. New York. Harper and Row Publ. Inc.

Hofkirchner, W. (1998) Emergence and the logic of explanation; An argument for the unity of science. In: Acta Polytechnica Scandinavica, Mathematics, Computing and Management in Engineering Series 91, pp- 23-30.

Ne'eman, Y. (1969) The Arrows of Time. In: Proceedings of the Israel Academy of Sciences \& Humanities, Section of Sciences, Hebrew version, Jerusalem, No. 13, pp. 1-13.

Ne'eman, Y. (1970) CP and CPT Symmetry Violations, Entropy and the Expanding Universe. In: Intern. J. of Theor. Phys. 3, pp- 1-6. Also pub. as Cosmological Implications of the Microscopic Time-Arrow, In: Relativity and Gravitation, Proc. of the Rosen Intern. Seminar, Haifa 1969, C. G. Kuper and A. Peres (Eds), London. Gordon and Breach, pp. 259-264.

Ne'eman, Y. (2003) The direction of time: Seven arrows for one stream? A program In: International Journal of Theoretical Physics, 42 (10): pp. 2557-2562.

Rosen, J. (1995) Symmetry in Science, New York. Springer-Verlag, 213 p. 